\title{
Diastereoselective addition of heteroatom nucleophiles to ethyl 2-(diethoxymethyl)cycloprop-2-ene-1-carboxylate
}

\author{
Angeles Martínez-Grau,* a José M. Blasco, ${ }^{a}$ Rafael Ferritto, ${ }^{a}$ Juan F. Espinosa, ${ }^{\text {a }}$ \\ Susana Mantecón, ${ }^{b}$ and Juan J.Vaquero* b \\ ${ }^{a}$ Centro de Investigación Lilly, Avda. de la Industria 30, 28108-Alcobendas, Madrid, Spain \\ ${ }^{b}$ Departamento de Química Orgánica, Facultad de Farmacia, Universidad de Alcalá de \\ Henares, 28871-Alcalá de Henares, Madrid, Spain \\ E-mail: juanjose.vaquero@uah.es
}

\section{Dedicated to Prof. José Elguero on the occasion of his $70^{\text {th }}$ birthday and to Prof. Pedro Molina on the occasion of his $60^{\text {th }}$ birthday}

(received 16 Dec 04; accepted 16 Jun 05; published on the web 21 Jun 05)

\begin{abstract}
We report a simple and straightforward route to hetero-functionalized cyclopropanes by addition of heteroatom nucleophiles to ethyl 2-(diethoxymethyl)cycloprop-2-ene-1-carboxylate. The resulting donor-acceptor substituted cyclopropane are useful synthetic intermediates owing to their high functionalization. Particular emphasis is placed in the stereochemical outcome of this reaction.
\end{abstract}

Keywords: Cyclopropanes, nucleophilic addition, heteroatom nucleophiles, ethyl 2(diethoxymethyl)cycloprop-2-ene-1-carboxylate

\section{Introduction}

Cyclopropenation of alkynes by diazocarbonyl compounds, promoted by copper or rhodium (II) catalysts, has been extensively studied. ${ }^{1}$ Rhodium (II) carboxylates and carboxamides have been found the most effective cyclopropenation catalysts, although the yields depend on the nature of the alkyne substituents. ${ }^{2}$ This reaction offers a direct entry to highly functionalized cyclopropenes, ${ }^{3}$ a very attractive class of strained rings that can be transformed into a wide variety of compounds because of its high reactivity. ${ }^{4}$ In the intramolecular version, the reaction of rhodium carbene complexes onto alkynes yields vinyl carbenoids that can be trapped in situ. $^{5}$ 


\section{Results and Discussion}

Following our work on the preparation of synthetically useful strained-ring organic molecules, we became interested in cyclopropanes of general structure A (Scheme 1). Owing to the absence of direct cyclopropanation methods for such hetero-substituted cyclopropanes, ${ }^{6}$ we anticipated that these compounds could be prepared by reaction of ethyl 2-(diethoxymethyl)cycloprop-2ene-1-carboxylate (1) with different heteroatom nucleophiles. In fact, the cyclopropene $\pi$-bond is very activated towards nucleophilic ${ }^{7}$ or electrophilic ${ }^{8}$ addition because these processes reduce considerably the ring strain, thus affording highly functionalized cyclopropanes. Carbometalation across the double bond, coupled with electrophilic trapping is probably the most frequently reported nucleophilic addition. ${ }^{9}$ However, the reaction of cyclopropenes containing non-conjugated electron-withdrawing substituents with soft nucleophiles has received less attention. ${ }^{10}$

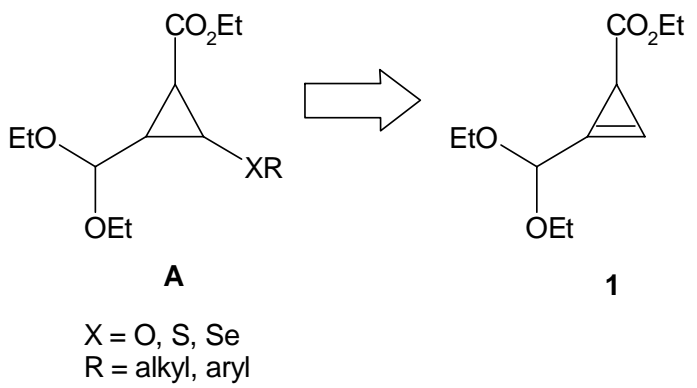

\section{Scheme 1}

Cyclopropenyl esters are very useful synthetic intermediates, readily available by slow addition of alkyl diazoacetates to an excess of the appropriate alkyne in the presence of a catalytic amount of rhodium acetate. $^{2}$ Following this general procedure, ethyl 2(diethoxymethyl)cycloprop-2-ene-1-carboxylate (1) was prepared in $72 \%$ yield by reaction of propionaldehyde diethyl acetal (2) with ethyl diazoacetate (Scheme 2), using dirhodium(II) tetraacetate as catalyst. The cyclopropene 1 was purified by column chromatography and could be kept in the freezer under nitrogen for several days. The enantioselective cyclopropenation of propionaldehyde diethyl acetal (2) with methyl diazoacetate, using $\mathrm{Rh}_{2}(5 S \text {-MEPY })_{4}$ as chiral catalyst, has been described previously, in $42 \%$ yield. ${ }^{11}$ In a more recent paper, this reaction has been optimized by increasing the addition time and the reagent ratio. ${ }^{12}$ 


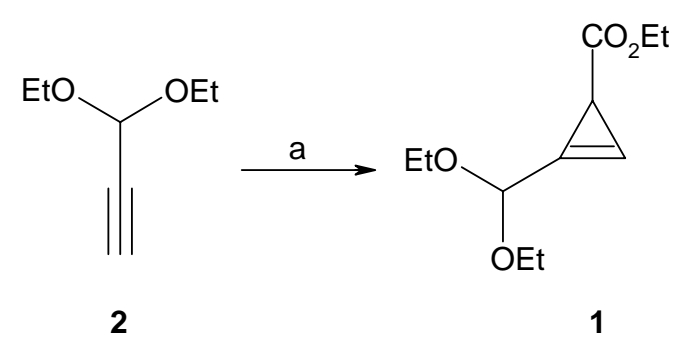

Scheme 2. (a) $\mathrm{Rh}_{2}(\mathrm{OAc})_{4}, \mathrm{~N}_{2} \mathrm{CHCO}_{2} \mathrm{Et}, \mathrm{CH}_{2} \mathrm{Cl}_{2}$, R.T., 24 h, $72 \%$.

With the cyclopropene 1 in hand we have examined the addition of heteroatom nucleophiles (Table 1). For this purpose, we chose thioalkoxides (entries 1-4), selenides (entries 5, 6), and alkoxides. Of the three species studied, thioalkoxides and selenides were found to be more suitable nucleophiles for the desired reaction. We attribute this result to their softer nucleophilicity and to the higher stability of the resulting cyclopropanes. As an example, when we attempted the reaction of cyclopropene 1 with sodium ethoxide in ethanol, the corresponding addition product was detected and isolated in very low yield (12\%). The same reaction with sodium methoxide in methanol provided the addition plus transesterification compound in $14 \%$ yield. A substantial amount of starting material was recovered in both cases.

The reactions proceeded under very mild conditions, at room temperature, using one equivalent of nucleophile. Acetonitrile was found to be the best solvent for thioalkoxides (entries 1-4) and DMF for selenides (entries 5 and 6). In all cases, a mixture of diastereomeric cyclopropanes 3 and 4 was obtained (Table 1). We were not able to separate the diastereomers by column chromatography, but they could be separated by HPLC. The relative configuration of cyclopropanes $\mathbf{3}$ and $\mathbf{4}$ was established by NMR spectroscopy, the assignment of the resonances being achieved through a combination of COSY and HMBC experiments. The stereochemistry of the cyclopropane ring was assigned from the observed coupling constants.

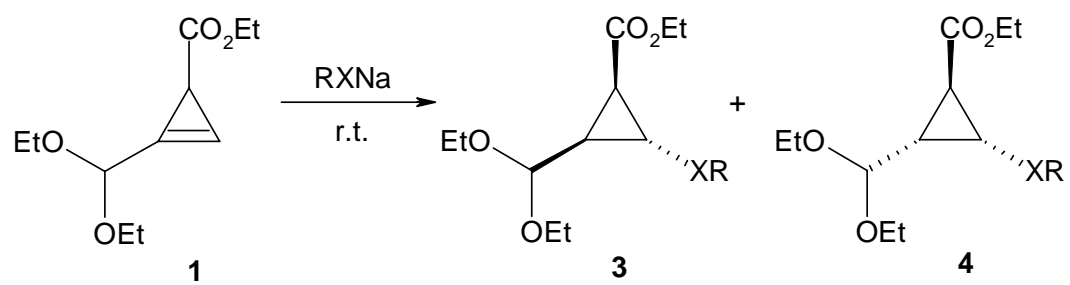


Table 1. Addition of heteroatom nucleophiles on ethyl 2-(diethoxymethyl)cycloprop-2-ene-1carboxylate (1)

\begin{tabular}{|c|c|c|c|c|c|c|}
\hline Entry & Compound & $\mathrm{X}$ & $\mathrm{R}$ & Solvent & Yield $^{\mathrm{a}}(\%)$ & 3:4 Ratio $^{b}$ \\
\hline 1 & $3 a, 4 a$ & $\mathrm{~S}$ & $\mathrm{Me}$ & $\mathrm{CH}_{3} \mathrm{CN}$ & 54 & $80: 20$ \\
\hline 2 & $3 b, 4 b$ & $\mathrm{~S}$ & $\mathrm{Et}$ & $\mathrm{CH}_{3} \mathrm{CN}$ & 50 & $92: 8$ \\
\hline 3 & $3 c, 4 c$ & $\mathrm{~S}$ & $\mathrm{CH}_{2} \mathrm{CO}_{2} \mathrm{Et}$ & THF & $25^{\mathrm{c}}$ & 100:0 \\
\hline 4 & 3d, 4d & $\mathrm{S}$ & $\mathrm{Ph}$ & $\mathrm{CH}_{3} \mathrm{CN}$ & 37 & $5: 95$ \\
\hline 5 & $3 e, 4 e$ & $\mathrm{Se}$ & $\mathrm{Me}$ & $\mathrm{DMF}$ & 48 & $22: 78$ \\
\hline 6 & $3 f, 4 f$ & $\mathrm{Se}$ & $\mathrm{Ph}$ & $\mathrm{DMF}$ & 58 & $5: 95$ \\
\hline
\end{tabular}

${ }^{a}$ Isolated overall yield after column chromatography.

${ }^{\mathrm{b}}$ Determined by ${ }^{1} \mathrm{H}-\mathrm{NMR}$.

${ }^{\mathrm{c}}$ The cyclopropane 5 (Figure 1) was isolated as the major compound, in $36 \%$ yield.

The stereoselectivity obtained in the formation of major isomers could be rationalized by assuming attack of the heteroatom nucleophile from the less sterically hindered side, trans- to the alkoxycarbonyl group. For the small XR groups (entries 1-3), the subsequent protonation could occur from the bottom side, yielding the cyclopropanes $\mathbf{3 a - 3 c}$ as major isomers. Bulkier groups (entries 4-6) should direct protonation from the top face, giving compounds $\mathbf{4 d - 4 f}$.

Although yields are moderate (Table 1), all the attempts to increase them were unsuccessful after an extensive search for optimal conditions. In most cases, no reaction was observed at $0{ }^{\circ} \mathrm{C}$. Heating of the reactions to $40{ }^{\circ} \mathrm{C}$ led to decomposition of the cyclopropene 1 . The use of a twofold molar excess of sodium thioalkoxide did not modify the yield.

For the same nucleophile type, we have observed that the yield decreases with the size of the $\mathrm{R}$ group (Table 1). In fact, when we tried the reaction with the sterically hindered sodium salt of 1methyl-5-mercaptotetrazole, no addition was observed. With sodium methylselenide (entry 5), the yield of the resulting cyclopropenes 3e, 4e was lower than expected, owing to some instability detected during purification. Although the cyclopropanes 3, 4 were stable and could be purified by chromatography, they were kept in the freezer under nitrogen to avoid slow decomposition. It is known that donor-acceptor substituted cyclopropanes are particularly unstable substrates, and they are prone to rapid ring-opening reactions. ${ }^{13}$

The reaction of ethyl 2-mercaptoacetate (entry 3) with cyclopropene 1 using sodium hydride as base (1.1 equiv.) provided the cyclopropane 3c only in $25 \%$ yield. This is due to the presence of the unexpected regioisomer 5 (Figure 1), which was isolated in $36 \%$ yield. Although the mechanism of formation of the cyclopropane 5 is unclear, its formation could be explained in terms of a process that involves partial isomerization of the double bond under the reaction 
conditions, and subsequent Michael addition. We attribute this competitive reaction to the lower nucleophilicity of ethyl 2-mercaptoacetate.

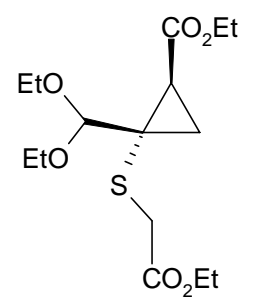

5

\section{Figure 1}

The relative configuration of the cyclopropane 5 was determined by NMR spectroscopy through a combination of COSY, HSQC and HMBC experiments. 1D- and 2D-NOESY experiments were carried out to determine the relative disposition of the substituents in the cyclopropane ring. The key NOE for determining the relative configuration is shown in Figure 2. The existence of a NOE between $\mathrm{H}-4$ and H-3a, although no NOEs were detected between H-4 and $\mathrm{H}-3 \mathrm{~b}$ or between $\mathrm{H}-4$ and H-1, demonstrates that $\mathrm{H}-4$ and $\mathrm{H}-3 \mathrm{a}$ are on the same side of the ring.

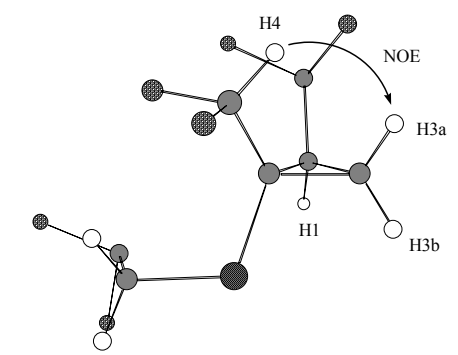

Figure 2. Minimum energy conformation of 5 (MMFF force field) showing the key NOE interaction. Ethyl groups are omitted for clarity.

\section{Conclusions}

In summary, we have shown that 3-hetero-substituted 2-(diethoxymethyl)cyclopropane-1carboxylates can be obtained easily, using a simple and convenient methodology, by addition of heteroatom nucleophiles to the readily available ethyl 2-(diethoxymethyl)cycloprop-2-ene-1carboxylate. Although yields were moderate, all reactions proceeded with high selectivity with a common trans- nucleophilic addition. The relative configuration of the third stereogenic center formed can be reversed by modifying the size of the nucleophile. 


\section{Experimental Section}

General Procedures. All solvents and reagents were purchased from commercial sources and used without further purification. All reactions were carried out under a positive pressure of nitrogen. RT denotes room temperature. Analytical TLC was performed on Merck TLC glass plates precoated with $\mathrm{F}_{254}$ silica gel 60. Silica gel 60 (Merck, 230-400 mesh) was used for flash chromatography. IR spectra were obtained on a Nicolet 510 P-FT. ${ }^{1} \mathrm{H}$ - and ${ }^{13} \mathrm{C}$ - NMR spectra were recorded on Bruker Avance 200 and 500 spectrometers as noted below. Chemical shifts are expressed as $\delta$ values ( $\mathrm{ppm})$ relative to internal TMS (0 ppm). Absolute-value COSY, phasesensitive HSQC, and HMBC spectra were acquired using gradient-selection techniques. 1DNOESY experiments were carried out with the selective 1D-double-pulse field gradient spin echo module using a mixing time of $500 \mathrm{~ms}$. 2D-NOESY experiments were also performed with the same mixing time. Data were processed using the XWINNMR Bruker program on a Silicon Graphics computer.

Ethyl 2-(diethoxymethyl)cycloprop-2-ene-1-carboxylate (1). To a solution of propionaldehyde diethyl acetal (2) (5.12 g, $40 \mathrm{mmol})$ and dirhodium(II) tetra-acetate $(0.177 \mathrm{~g}, 0.4 \mathrm{mmol})$ in anhydrous $\mathrm{CH}_{2} \mathrm{Cl}_{2}(60 \mathrm{~mL})$, was added ethyl diazoacetate $(2.1 \mathrm{~mL}, 20 \mathrm{mmol})$ in anhydrous $\mathrm{CH}_{2} \mathrm{Cl}_{2}(60 \mathrm{~mL})$, via syringe pump, during $27 \mathrm{~h}$, at RT under nitrogen. After addition was complete, the solvent was removed under vacuum. Hexane was added to the resulting oil, and the insoluble catalyst removed by filtration through Celite. The filtrate was concentrated, and the crude residue submitted to flash chromatography (silica gel pretreated with $1 \%$ of $\mathrm{Et}_{3} \mathrm{~N}$, hexane/ethyl acetate, 9:1) to give the title compound as an oil (3.08 g, 72\% yield). IR $\left(\mathrm{CCl}_{4}\right) \mathrm{v}$ 3138, 2978, 2934, 2899, 1728, 1447, 1371, 1340, 1132, 1117, $1081 \mathrm{~cm}^{-1} .{ }^{1} \mathrm{H}-\mathrm{NMR}\left(\mathrm{CDCl}_{3}, 200\right.$ $\mathrm{MHz}) \delta 6.80(\mathrm{t}, J=1.1 \mathrm{~Hz}, 1 \mathrm{H}, \mathrm{CH}=\mathrm{C}), 5.51\left[\mathrm{~d}, J=1.1 \mathrm{~Hz}, 1 \mathrm{H}, \mathrm{CH}(\mathrm{OEt})_{2}\right], 4.14\left(\mathrm{q}, 2 \mathrm{H}, \mathrm{CH}_{2}\right)$, 3.80-3.57 (m, 4H, $2 \mathrm{CH}_{2}$ ), 2.37 (d, J = 1.3 Hz, 1H, CH), 1.26 (t, 3H, $\left.\mathrm{CH}_{3}\right), 1.24$ (t, 3H, $\mathrm{CH}_{3}$ ), $1.23\left(\mathrm{t}, 3 \mathrm{H}, \mathrm{CH}_{3}\right) ;{ }^{13} \mathrm{C} \mathrm{NMR}\left(\mathrm{CDCl}_{3}, 50 \mathrm{MHz}\right) \delta 174.98\left(\mathrm{CO}_{2} \mathrm{Et}\right), 112.82(\mathrm{C}=\mathrm{CH}), 100.38$ $(\mathrm{C}=\mathrm{CH}), 95.74\left[\mathrm{CH}(\mathrm{OEt})_{2}\right], 61.54\left(\mathrm{CH}_{2}\right), 61.24\left(\mathrm{CH}_{2}\right), 60.36\left(\mathrm{CH}_{2}\right), 20.83(\mathrm{CH}), 15.06\left(2 \mathrm{CH}_{3}\right)$, $14.24\left(\mathrm{CH}_{3}\right)$; MS m/z $214\left(\mathrm{M}^{+}, 2\right), 185$ (11), 169 (22), 141 (54), 113 (100), 103 (70), 85 (72), 75 (70). Anal. Calcd for $\mathrm{C}_{11} \mathrm{H}_{18} \mathrm{O}_{4}$ : C, 61.66; H, 8.47. Found: C, 61.36; H, 8.37\%.

\section{Reaction of ethyl 2-(diethoxymethyl)cycloprop-2-ene-1-carboxylate (1) with sodium thiomethoxide}

To a solution of ethyl 2-(diethoxymethyl)cycloprop-2-ene-1-carboxylate (1) $(0.214 \mathrm{~g}, 1 \mathrm{mmol})$ in anhydrous acetonitrile $(5 \mathrm{~mL})$ was added sodium thiomethoxide $(77 \mathrm{mg}, 1.1 \mathrm{mmol})$ at RT under $\mathrm{N}_{2}$. The reaction mixture was stirred at RT for 2 hours. The solvent was removed (vacuum) and the residue partitioned between ethyl acetate and water. The organic phase was dried over $\mathrm{MgSO}_{4}$, filtered and concentrated. The crude mixture was submitted to flash chromatography (hexane/ethyl acetate, 9:1) isolating a mixture of compounds 3a/4a (80:20 ratio, 54\% yield). Compound 3a was purified by HPLC for characterization. 
Ethyl (1SR,2RS,3SR)-2-diethoxymethyl-3-(methylsulfanyl)cyclopropane-1-carboxylate (3a). Colorless oil. IR $\left(\mathrm{CCl}_{4}\right)$ v 2978, 2922, 1728, 1441, 1398, 1379, 1350, 1321, 1194, 1117 , $1082 \mathrm{~cm}^{-1} .{ }^{1} \mathrm{H}-\mathrm{NMR}\left(\mathrm{CDCl}_{3}, 200 \mathrm{MHz}\right) \delta 4.51\left[\mathrm{~d}, J=7.7 \mathrm{~Hz}, 1 \mathrm{H}, \mathrm{CH}(\mathrm{OEt})_{2}\right], 4.14(\mathrm{q}, 2 \mathrm{H}$, $\mathrm{CH}_{2}$ ), 3.75-3.35 (m, 4H, $\left.2 \mathrm{CH}_{2}\right), 2.66(\mathrm{dd}, J=4.6,5.7 \mathrm{~Hz}, 1 \mathrm{H}, \mathrm{H}-3), 2.17\left(\mathrm{~s}, 3 \mathrm{H}, \mathrm{SCH}_{3}\right), 1.95$ (dd, $J=4.6,9.6 \mathrm{~Hz}, 1 \mathrm{H}, \mathrm{H}-1), 1.83$ (ddd, $J=5.7,7.7,9.6 \mathrm{~Hz}, 1 \mathrm{H}, \mathrm{H}-2), 1.27$ (t, 3H, $\left.\mathrm{CH}_{3}\right), 1.22$ $\left(\mathrm{t}, 3 \mathrm{H}, \mathrm{CH}_{3}\right), 1.14\left(\mathrm{t}, 3 \mathrm{H}, \mathrm{CH}_{3}\right) ;{ }^{13} \mathrm{C}-\mathrm{NMR}\left(\mathrm{CDCl}_{3}, 50 \mathrm{MHz}\right) \delta 170.73\left(\mathrm{CO}_{2} \mathrm{Et}\right), 100.18$ $\left[\mathrm{CH}(\mathrm{OEt})_{2}\right], 61.80\left(\mathrm{CH}_{2}\right), 61.54\left(\mathrm{CH}_{2}\right), 60.89\left(\mathrm{CH}_{2}\right), 33.75(\mathrm{CH}), 27.43(\mathrm{CH}), 27.38(\mathrm{CH}), 16.22$ $\left(\mathrm{CH}_{3}\right), 15.43\left(\mathrm{CH}_{3}\right), 15.17\left(\mathrm{CH}_{3}\right), 14.19\left(\mathrm{CH}_{3}\right) . \mathrm{MS}$ m/z $217\left(\mathrm{M}^{+}-45,40\right), 189$ (4), 171 (16), 159 (17), 143 (17), 131 (14), 115 (19), 103 (100), 75 (61). Anal. Calcd for $\mathrm{C}_{12} \mathrm{H}_{22} \mathrm{O}_{4} \mathrm{~S}$ : C, 54.94; H, 8.45. Found: C, 54.62; H, 8.73\%.

\section{Reaction of ethyl 2-(diethoxymethyl)cycloprop-2-ene-1-carboxylate (1) with sodium} thioethoxide

To a solution of ethanethiol $(0.068 \mathrm{~g}, 1.1 \mathrm{mmol})$ in anhydrous acetonitrile $(2 \mathrm{~mL})$ was added sodium hydride $(1.1 \mathrm{mmol})$ under $\mathrm{N}_{2}$. After stirring for $30 \mathrm{~min}$, a solution of $\mathbf{1}(0.214 \mathrm{~g}, 1 \mathrm{mmol})$ in anhydrous acetonitrile $(3 \mathrm{~mL})$ was added at $\mathrm{RT}$. The reaction mixture was stirred at RT for $3 \mathrm{~h}$. The solvent was removed (vacuum) and the residue partitioned between ethyl acetate and water. The organic phase was dried over $\mathrm{MgSO}_{4}$, filtered and concentrated. The crude mixture was submitted to flash chromatography (hexane/ethyl acetate, 9:1), giving a mixture of compounds $\mathbf{3 b} / \mathbf{4 b}$ (92:8 ratio, 50\% yield). Compound $\mathbf{3 b}$ was purified by HPLC for characterization.

Ethyl (1SR,2RS,3SR)-2-diethoxymethyl-3-(ethylsulfanyl)cyclopropane-1-carboxylate (3b). Colorless oil. IR $\left(\mathrm{CCl}_{4}\right)$ v 2978, 2922, 1728, 1441, 1398, 1379, 1350, 1321, 1194, 1117 , $1082 \mathrm{~cm}^{-1} .{ }^{1} \mathrm{H}-\mathrm{NMR}\left(\mathrm{CDCl}_{3}, 200 \mathrm{MHz}\right) \delta 4.54\left[\mathrm{~d}, J=7.8 \mathrm{~Hz}, 1 \mathrm{H}, \mathrm{CH}(\mathrm{OEt})_{2}\right], 4.14(\mathrm{q}, 2 \mathrm{H}$, $\mathrm{CH}_{2}$ ), 3.78-3.38 (m, 4H, $\left.2 \mathrm{CH}_{2}\right), 2.66$ (dd, J=4.6, 5.7 Hz, 1H, H-3), 2.62 (q, 2H, $\mathrm{CH}_{2}$ ), 1.95 (dd, $J=4.6,9.6 \mathrm{~Hz}, 1 \mathrm{H}, \mathrm{H}-1), 1.82(\mathrm{ddd}, J=5.7,7.8,9.6 \mathrm{~Hz}, 1 \mathrm{H}, \mathrm{H}-2), 1.36-1.12\left(4 \mathrm{t}, 12 \mathrm{H}, 4 \mathrm{CH}_{3}\right)$; ${ }^{13} \mathrm{C}-\mathrm{NMR}\left(\mathrm{CDCl}_{3}, 50 \mathrm{MHz}\right) \delta 170.78\left(\mathrm{CO}_{2} \mathrm{Et}\right), 100.26\left[\mathrm{CH}(\mathrm{OEt})_{2}\right], 61.82\left(\mathrm{CH}_{2}\right), 61.12\left(\mathrm{CH}_{2}\right)$, $60.88\left(\mathrm{CH}_{2}\right), 33.51(\mathrm{CH}), 27.49(\mathrm{CH}), 27.24(\mathrm{CH}), 25.31\left(\mathrm{SCH}_{2}\right), 15.41\left(\mathrm{CH}_{3}\right), 15.22\left(\mathrm{CH}_{3}\right)$, $14.72\left(\mathrm{CH}_{3}\right), 14.22\left(\mathrm{CH}_{3}\right)$. Anal. Calcd for $\mathrm{C}_{13} \mathrm{H}_{24} \mathrm{O}_{4} \mathrm{~S}: \mathrm{C}, 56.49 ; \mathrm{H}, 8.75$. Found: C, 56.26; H, $8.37 \%$.

Reaction of ethyl 2-(diethoxymethyl)cycloprop-2-ene-1-carboxylate (1) with ethyl 2mercaptoacetate

To a solution of ethyl 2-mercaptoacetate $(0.132 \mathrm{~g}, 1.1 \mathrm{mmol})$ in anhydrous THF $(2 \mathrm{~mL})$ was added sodium hydride $(1.1 \mathrm{mmol})$ under $\mathrm{N}_{2}$. After stirring for $1 \mathrm{~h}$, a solution of $1(0.214 \mathrm{~g}$, $1 \mathrm{mmol})$ in anhydrous THF $(3 \mathrm{~mL})$ was added at RT. The reaction mixture was stirred at RT for $14 \mathrm{~h}$. Water was added and the mixture extracted twice with ethyl acetate. The organic phase was dried over $\mathrm{MgSO}_{4}$, filtered and concentrated. The crude mixture was submitted to flash chromatography (hexane/ethyl acetate, 4:1) isolating compounds 3c (97 mg, 25\% yield) and 5 (140 mg, $36 \%$ yield). 
Ethyl (1SR,2RS,3SR)-2-diethoxymethyl-3-(ethoxycarbonylmethylsulfanyl)cyclopropane-1carboxylate (3c). Colorless oil. IR $\left(\mathrm{CCl}_{4}\right)$ v 2978, 2934, 2903, 1730, 1445, 1379, 1271, 1194, 1116, 1059, $1032 \mathrm{~cm}^{-1} .{ }^{1} \mathrm{H}-\mathrm{NMR}\left(\mathrm{CDCl}_{3}, 200 \mathrm{MHz}\right) \delta 4.51\left[\mathrm{~d}, J=7.8 \mathrm{~Hz}, 1 \mathrm{H}, \mathrm{CH}(\mathrm{OEt})_{2}\right]$, 4.26-4.08 (m, 4H, $2 \mathrm{CH}_{2}$ ), 3.75-3.37 (m, 4H, $2 \mathrm{CH}_{2}$ ), 3.32 (d, $J=1.9 \mathrm{~Hz}, 2 \mathrm{H}, \mathrm{CH}_{2}$ ), 2.83 (dd, $J$ $=4.6,5.9 \mathrm{~Hz}, 1 \mathrm{H}, \mathrm{H}-3), 2.01(\mathrm{dd}, J=4.6,9.7 \mathrm{~Hz}, 1 \mathrm{H}, \mathrm{H}-1), 1.87(\mathrm{ddd}, J=5.6,7.8,9.7 \mathrm{~Hz}, 1 \mathrm{H}$, $\mathrm{H}-2), 1.28\left(\mathrm{t}, 3 \mathrm{H}, \mathrm{CH}_{3}\right), 1.27\left(\mathrm{t}, 3 \mathrm{H}, \mathrm{CH}_{3}\right), 1.22\left(\mathrm{t}, 3 \mathrm{H}, \mathrm{CH}_{3}\right), 1.14\left(\mathrm{t}, 3 \mathrm{H}, \mathrm{CH}_{3}\right) ;{ }^{13} \mathrm{C}-\mathrm{NMR}$ $\left(\mathrm{CDCl}_{3}, 50 \mathrm{MHz}\right) \delta 170.2\left(\mathrm{CO}_{2} \mathrm{Et}\right), 169.8\left(\mathrm{CO}_{2} \mathrm{Et}\right), 100.0\left[\mathrm{CH}(\mathrm{OEt})_{2}\right], 62.1\left(\mathrm{CH}_{2}\right), 61.4\left(2 \mathrm{CH}_{2}\right)$, $60.9\left(\mathrm{CH}_{2}\right), 35.2\left(\mathrm{CH}_{2} \mathrm{~S}\right), 33.5(\mathrm{CH}), 27.6(\mathrm{CH}), 26.0(\mathrm{CH}), 15.3\left(\mathrm{CH}_{3}\right), 15.1\left(\mathrm{CH}_{3}\right), 14.1\left(\mathrm{CH}_{3}\right)$, $14.0\left(\mathrm{CH}_{3}\right)$; MS (Electrospray) $357.1\left(\mathrm{M}^{+}+\mathrm{Na}\right)$. Anal. Calcd for $\mathrm{C}_{15} \mathrm{H}_{26} \mathrm{O}_{6} \mathrm{~S}: \mathrm{C}, 53.87 ; \mathrm{H}, 7.84$. Found: C, 54.11; H, 8.07\%.

Ethyl (1SR,2SR)-2-diethoxymethyl-2-(ethoxycarbonylmethylsulfanyl)cyclopropane1-carboxylate (5). Oil. IR $\left(\mathrm{CCl}_{4}\right)$ v 2980, 2934, 2905, 1728, 1447, 1400, 1379, 1348, 1203, $1159,1111,1067,1030 \mathrm{~cm}^{-1} .{ }^{1} \mathrm{H}-\mathrm{NMR}\left(\mathrm{CDCl}_{3}, 500 \mathrm{MHz}\right) \delta 4.50\left[\mathrm{~s}, 1 \mathrm{H}, \mathrm{CH}(\mathrm{OEt})_{2}\right], 4.17$ (q, $\left.2 \mathrm{H}, \mathrm{CH}_{2}\right), 4.13\left(\mathrm{q}, 2 \mathrm{H}, \mathrm{CH}_{2}\right), 3.75-3.57(\mathrm{~m}, 5 \mathrm{H}), 3.37-3.29(\mathrm{~m}, 1 \mathrm{H}), 2.24(\mathrm{dd}, J=6.4,8.5 \mathrm{~Hz}$, $1 \mathrm{H}, \mathrm{H}-1), 1.60$ (dd, $J=5.4,6.4 \mathrm{~Hz}, 1 \mathrm{H}, \mathrm{H}-3 \mathrm{a}), 1.50$ (dd, $J=5.4,8.5 \mathrm{~Hz}, 1 \mathrm{H}, \mathrm{H}-3 \mathrm{~b}), 1.27$ (t, 3H, $\left.\mathrm{CH}_{3}\right), 1.26\left(\mathrm{t}, 3 \mathrm{H}, \mathrm{CH}_{3}\right), 1.24\left(\mathrm{t}, 3 \mathrm{H}, \mathrm{CH}_{3}\right), 1.13\left(\mathrm{t}, 3 \mathrm{H}, \mathrm{CH}_{3}\right) ;{ }^{13} \mathrm{C}-\mathrm{NMR}\left(\mathrm{CDCl}_{3}, 125 \mathrm{MHz}\right) \delta$ 170.7, $170.6\left(2 \mathrm{CO}_{2} \mathrm{Et}\right), 104.3\left[\mathrm{CH}(\mathrm{OEt})_{2}\right], 64.6\left(\mathrm{CH}_{2}\right), 64.0\left(\mathrm{CH}_{2}\right), 61.4\left(\mathrm{CH}_{2}\right), 61.1\left(\mathrm{CH}_{2}\right), 36.8$ (C-2), $36.2\left(\mathrm{SCH}_{2}\right), 28.2(\mathrm{C}-1), 21.4(\mathrm{C}-3), 15.6\left(\mathrm{CH}_{3}\right), 15.3\left(\mathrm{CH}_{3}\right), 14.3\left(\mathrm{CH}_{3}\right), 14.2\left(\mathrm{CH}_{3}\right)$; $\mathrm{MS}$ (Electrospray) $357.2\left(\mathrm{M}^{+}+\mathrm{Na}\right)$. Anal. Calcd for $\mathrm{C}_{15} \mathrm{H}_{26} \mathrm{O}_{6} \mathrm{~S}: \mathrm{C}, 53.87$; H, 7.84. Found: C, 53.91; $\mathrm{H}, 8.11 \%$.

\section{Reaction of ethyl 2-(diethoxymethyl)cycloprop-2-ene-1-carboxylate (1) with sodium thiophenoxide}

To a solution of $1(0.214 \mathrm{~g}, 1 \mathrm{mmol})$ in anhydrous acetonitrile $(5 \mathrm{~mL})$, sodium thiophenoxide $(0.145 \mathrm{~g}, 1.1 \mathrm{mmol})$ was added at RT under $\mathrm{N}_{2}$. The reaction mixture was stirred at room temperature for $2 \mathrm{~h}$, the solvent removed under vacuum, and the residue partitioned between ethyl acetate and water. The organic phase was dried over $\mathrm{MgSO}_{4}$, filtered and concentrated. The crude mixture was submitted to flash chromatography (hexane/ethyl acetate, 9:1), isolating a mixture of compounds $\mathbf{3 d} / \mathbf{4 d}$ (5:95 ratio, 37\% yield).

Ethyl (1SR,2SR,3SR)-2-diethoxymethyl-3-(phenylsulfanyl)cyclopropane-1-carboxylate (4d). Colorless oil. IR $\left(\mathrm{CCl}_{4}\right)$ v 3102, 2978, 2930, 1728, 1682, 1457, 1389, 1195, $1070 \mathrm{~cm}^{-1}$. ${ }^{1} \mathrm{H}$ - NMR $\left(\mathrm{CDCl}_{3}, 200 \mathrm{MHz}\right) \delta 7.36-7.12\left(\mathrm{~m}, 5 \mathrm{H}\right.$, Aromatic), $4.53\left[\mathrm{~d}, J=7.5 \mathrm{~Hz}, 1 \mathrm{H}, \mathrm{CH}(\mathrm{OEt})_{2}\right], 4.20-$ $4.10\left(\mathrm{~m}, 2 \mathrm{H}, \mathrm{CH}_{2}\right), 3.72-3.35\left(\mathrm{~m}, 4 \mathrm{H}, 2 \mathrm{CH}_{2}\right), 2.93(\mathrm{dd}, J=4.5,8.4 \mathrm{~Hz}, 1 \mathrm{H}, \mathrm{H}-3), 2.27(\mathrm{~m}, J=$ 5.4, 7.5, 8.4 Hz, 1H, H-2), 1.94 (t, J=5.4 Hz, 1H, H-1), 1.26 (t, 3H, $\left.\mathrm{CH}_{3}\right), 1.20$ (t, 3H, $\mathrm{CH}_{3}$ ), $1.10\left(\mathrm{t}, 3 \mathrm{H}, \mathrm{CH}_{3}\right) ;{ }^{13} \mathrm{C}-\mathrm{NMR}\left(\mathrm{CDCl}_{3}, 50 \mathrm{MHz}\right) \delta 171.50\left(\mathrm{CO}_{2} \mathrm{Et}\right), 130.53,128.88,127.29$, 125.74 (Aromatics), $100.65\left[\mathrm{CH}(\mathrm{OEt})_{2}\right], 61.77\left(\mathrm{CH}_{2}\right), 61.21\left(\mathrm{CH}_{2}\right), 60.68\left(\mathrm{CH}_{2}\right), 30.71(\mathrm{CH})$, $26.82(\mathrm{CH}), 26.58(\mathrm{CH}), 15.43\left(\mathrm{CH}_{3}\right), 15.13\left(\mathrm{CH}_{3}\right), 14.26\left(\mathrm{CH}_{3}\right)$. Anal. Calcd for $\mathrm{C}_{17} \mathrm{H}_{24} \mathrm{O}_{4} \mathrm{~S}: \mathrm{C}$, 62.94; H, 7.46. Found: C, 63.31; H, 7.70\%. 
Reaction of ethyl 2-(diethoxymethyl)cycloprop-2-ene-1-carboxylate (1) with sodium selenides

To a solution of dimethyl diselenide or diphenyl diselenide $(0.55 \mathrm{mmol})$ in anhydrous DMF $(3 \mathrm{~mL})$ was added sodium borohydride $(0.042 \mathrm{~g}, 1.1 \mathrm{mmol})$ in portions. The mixture stirred for 1 $\mathrm{h}$ at RT until the solution was colorless. A solution of $1(0.214 \mathrm{~g}, 1 \mathrm{mmol})$ in anhydrous DMF $(2 \mathrm{~mL})$ was added and the reaction mixture stirred at RT for $24 \mathrm{~h}$. Water was added and the aqueous phase extracted twice with ethyl acetate. The organic phase was dried over $\mathrm{MgSO}_{4}$, filtered and concentrated. The crude mixture was submitted to flash chromatography (hexane/ethyl acetate, 4:1), isolating a mixture of compounds 3e/4e (22:78 ratio, 48\% yield) and 3f/4f (5:95 ratio, 58\% yield).

Ethyl (1SR,2SR,3SR)-2-diethoxymethyl-3-(methylselanyl)cyclopropane-1-carboxylate (4e). Colorless oil. IR $\left(\mathrm{CCl}_{4}\right)$ v 2978, 2922, 1728, 1441, 1398, 1379, 1350, 1321, 1194, 1117 , $1082 \mathrm{~cm}^{-1} .{ }^{1} \mathrm{H}-\mathrm{NMR}\left(\mathrm{CDCl}_{3}, 200 \mathrm{MHz}\right) \delta 4.51\left[\mathrm{~d}, J=7.8 \mathrm{~Hz}, 1 \mathrm{H}, \mathrm{CH}(\mathrm{OEt})_{2}\right], 4.22-4.10(\mathrm{~m}, 2 \mathrm{H}$, $\left.\mathrm{CH}_{2}\right), 3.80-3.58\left(\mathrm{~m}, 4 \mathrm{H}, 2 \mathrm{CH}_{2}\right), 2.68(\mathrm{dd}, J=4.8,9.0 \mathrm{~Hz}, 1 \mathrm{H}, \mathrm{H}-3), 2.11\left(\mathrm{~s}, 3 \mathrm{H}, \mathrm{SeCH}_{3}\right), 2.10$ $(\mathrm{m}, 1 \mathrm{H}, \mathrm{H}-2), 1.86(\mathrm{t}, J=4.8 \mathrm{~Hz}, 1 \mathrm{H}, \mathrm{H}-1), 1.32-1.20\left(3 \mathrm{t}, 9 \mathrm{H}, 3 \mathrm{CH}_{3}\right) ;{ }^{13} \mathrm{C}-\mathrm{NMR}\left(\mathrm{CDCl}_{3}\right.$, $50 \mathrm{MHz}) \delta 172.13\left(\mathrm{CO}_{2} \mathrm{Et}\right), 102.27\left[\mathrm{CH}(\mathrm{OEt})_{2}\right], 61.24,60.97\left(3 \mathrm{CH}_{2}\right), 30.25(\mathrm{CH}), 26.89(\mathrm{CH})$, $18.97(\mathrm{CH}), 15.46\left(\mathrm{CH}_{3}\right), 15.33\left(\mathrm{CH}_{3}\right), 14.18\left(\mathrm{CH}_{3}\right), 5.63\left(\mathrm{SeCH}_{3}\right) . \mathrm{C}_{12} \mathrm{H}_{22} \mathrm{O}_{4} \mathrm{Se}: \mathrm{C}, 46.61 ; \mathrm{H}$, 7.17. Found: C, 46.67; H, 6.93\%.

Ethyl (1SR,2SR,3SR)-2-diethoxymethyl-3-(phenylselanyl)cyclopropane-1-carboxylate (4f). Colorless oil. IR $\left(\mathrm{CCl}_{4}\right)$ v 3102, 2978, 2930, 1728, 1682, 1457, 1389, 1195, $1070 \mathrm{~cm}^{-1}$. ${ }^{1} \mathrm{H}-\mathrm{NMR}$ $\left(\mathrm{CDCl}_{3}, 200 \mathrm{MHz}\right) \delta 7.47$ (m, 2H, Aromatics), 7.25 (m, 3H, Aromatics), 4.53 [d, J=6.6 Hz, 1H, $\left.\mathrm{CH}(\mathrm{OEt})_{2}\right], 4.22-4.08\left(\mathrm{~m}, 2 \mathrm{H}, \mathrm{CH}_{2}\right), 3.72-3.42\left(\mathrm{~m}, 4 \mathrm{H}, 2 \mathrm{CH}_{2}\right), 2.98(\mathrm{dd}, J=4.8,9.0 \mathrm{~Hz}, 1 \mathrm{H}, \mathrm{H}-$ 3), $2.20(\mathrm{~m}, J=4.8,7.2,8.7 \mathrm{~Hz}, 1 \mathrm{H}, \mathrm{H}-2), 2.00$ (t, $J=4.8 \mathrm{~Hz}, 1 \mathrm{H}, \mathrm{H}-1), 1.25$ (t, 3H, $\left.\mathrm{CH}_{3}\right), 1.21$ $\left(\mathrm{t}, 3 \mathrm{H}, \mathrm{CH}_{3}\right), 1.15\left(\mathrm{t}, 3 \mathrm{H}, \mathrm{CH}_{3}\right) ;{ }^{13} \mathrm{C}-\mathrm{NMR}\left(\mathrm{CDCl}_{3}, 50 \mathrm{MHz}\right) \delta 171.88\left(\mathrm{CO}_{2} \mathrm{Et}\right), 130.82,129.08$, 126.64 (Aromatics), $101.93\left[\mathrm{CH}(\mathrm{OEt})_{2}\right], 61.23,61.04,60.97\left(3 \mathrm{CH}_{2}\right), 29.89(\mathrm{CH}), 26.86(\mathrm{CH})$, $20.52(\mathrm{CH}), 15.37,15.19,14.21\left(3 \mathrm{CH}_{3}\right)$. Anal. Calcd for $\mathrm{C}_{17} \mathrm{H}_{24} \mathrm{O}_{4} \mathrm{Se}: \mathrm{C}, 54.99 ; \mathrm{H}, 6.51$. Found: C, 54.73; H, 6.76\%.

\section{Acknowledgements}

Susana Mantecón thanks Eli Lilly \& Co. for fellowship support. José M. Blasco is grateful to the Ministerio de Educación y Cultura (Spain) for a doctoral research fellowship.

\section{References}

1. (a) Doyle, M. P.; McKervey, M. A.; Ye, T. Modern Catalytic Methods for Organic Synthesis with Diazo Compounds; John Wiley \& Sons: New York, 1998. (b) Doyle, M. P. In Comprehensive Organometallic Chemistry II; Abel, E. W.; Stone, F. G. A.; Wilkinson, G., 
Eds; Pergamon Press: Oxford, U. K., 1995; Vol. 12, p 387. (c) Protopopova, M. N.; Shapiro, E. A. Russ. Chem. Rev. 1989, 58, 667. (d) Doyle, M. P.; Protopopova, M. N.; Brandes, B. D.; Davies, H. M. L.; Huby, N. J. S.; Whitesell, J. K. Synlett 1993, 151. (e) Protopopova, M. N.; Doyle, M. P.; Müller, P.; Ene, D. J. Am. Chem. Soc. 1992, 114, 2755. (e) O'Bannon, P. E.; Dailey, W. P. J. Org. Chem. 1991, 56, 2258. (f). Maas, G. Top. Curr. Chem. 1987, 137, 75.

2. Petiniot, N.; Anciaux, A. J.; Noels, A. F.; Hubert, A. J.; Teyssié, P. Tetrahedron Lett. 1978, $14,1239$.

3. (a) Lebel, H.; Marcoux, J.-F.; Molinaro, C.; Charette, A. B. Stereoselective Cyclopropanation Reactions, Chem. Rev. 2003, 103, 977. (b) Halton, B.; Banwell, M. G. In The Chemistry of the Cyclopropyl Group; Rappoport, Z., Ed.; Wiley: New York, 1987, Part 2, Ch. 21, p 1223. (c) Baird, M. S. Top. Curr. Chem. 1988, 144, 137. (d) Binger, P.; Buech, H. M. Top. Curr. Chem. 1987, 135, 77.

4. (a) Cho, S. H.; Liebeskind, L. S. J. Org. Chem. 1987, 52, 2631. (b) Müller, P.; Gränicher, C. Helv. Chim. Acta 1993, 521. (c) Padwa, A.; Kulkarni, Y. S.; Terry, L. W. J. Org. Chem. 1990, 55, 2478. (d) Rigby, J. H.; Kierkus, P. C.; Head, D. Tetrahedron Lett. 1989, 30, 5073. (e) Franck-Neumann, M.; Miesch, M.; Kempf, H. Synthesis 1989, 820. (f) Padwa, A.; Krumpe, K. E.; Terry, L. W.; Wannamaker, M. W. J. Org. Chem. 1989, 54, 1635. (g) Binger, P.; Biedenbach, B. Chem. Ber. 1987, 120, 601. (h) Dowd, P.; Garner, P.; Schappert, R. J. Org. Chem. 1982, 47, 4240. (h) Dowd, P.; Schappert, R.; Garner, P.; Go, C. L. J. Org. Chem. 1985, 50, 44. (i) Müller, P.; Pautex, N.; Doyle, M. P.; Bagheri, V. Helv. Chim. Acta 1990, 73, 1233. (j) Al-Dulayymi, J.; Baird, M. S.; Pavlov, V. A.; Kurdjukov, A. I. Tetrahedron 1996, 52, 8877. (k) DeSimone, D. M.; Desrosiers, P. J.; Hughes, R. P. J. Am. Chem. Soc. 1982, 104, 4842. (1) Donaldson, W. A.; Hughes, R. P. J. Am. Chem. Soc. 1982, 104, 4846. (m) Rigby, J. H.; Kierkus, P. C. J. Am. Chem. Soc. 1989, 111, 4125.

5. (a) Padwa, A.; Austin, D. J.; Xu, S. L. J. Org. Chem. 1992, 57, 1330. (b) Padwa, A.; Krumpe, K. E.; Kassir, J. M. J. Org. Chem. 1992, 57, 4940. (c) Padwa, A.; Kinder, F. R. J. Org. Chem. 1993, 58, 21. (d) Padwa, A.; Kassir, J. M.; Xu, S. L. J. Org. Chem. 1997, 62, 1642. (e) Hoye, T. R.; Dinsmore, C. J. Tetrahedron Lett. 1992, 33, 169.

6. Reissig, H-U.; Zimmer, R. Donor-Acceptor Cyclopropane Derivatives and their Application in Organic Synthesis, Chem. Rev. 2003, 103, 1151.

7. (a) Shields, T. C.; Gardner, P. D. J. Am. Chem. Soc. 1967, 89, 5425. (b) Franck-Neumann, M.; Miesch, M.; Kempf, H. Tetrahedron 1988, 44, 2933. (c) Halton, B.; Banwell, M. G. In The Chemistry of the Cyclopropyl Group; Patai, S., Rappoport, Z., Eds; Wiley: Chichester, 1987; p 1224. (d) Baird, M. S. Top. Curr. Chem. 1988, 144, 139. (e) Baird, M. S.; Schmidt, T. In Carbocyclic Three-Membered Ring Compounds; de Meijere, Ed.; Georg Thieme Verlag: Stuttgart, 1996; p 114. (f) Nakamura, M.; Hirai, A.; Nakamura, E. J. Am. Chem. Soc. 2000, 122, 978 and refs. therein. (g) Nakamura, M.; Inoue, T.; Sato, A.; Nakamura, E. Org. Lett. 2000, 2, 2193 and refs. therein. (h) Richey, H. G., Jr.; Bension, R. M. J. Org. Chem. 1980, 45, 5036. (i) Araki, S.; Nakano, H.; Subburaj, K.; Hirashita, T.; Shibutani, K.; 
Yamamura, H.; Kawai, M.; Butsugan, Y. Tetrahedron Lett. 1998, 39, 6327. (j) Araki, S.; Shiraki, F.; Tanaka, T.; Nakano, H.; Subburaj, K.; Hirashita, T.; Yamamura, H.; Kawai, M. Chem.-Eur. J. 2001, 7, 2784.

8. (a) Baird, M. S.; Shortt, M. F.; Hussain, H. H.; Al Dulayymi, J. R. J. Chem. Soc,. Perkin Trans. 1 1993, 17, 1945. (b) Kartashov, V. R.; Skorobogatova, E. V.; Afanas'ev, P. S.; Chertkov, V. A.; Sergeev, N. M. Chernov. A. N.; Zefirov, N. S. Zh. Org. Khim. 1991, 27, 1861. (c) Kartashov, V. R.; Skorobogatova, E. V.; Vasileva, O. V.; Malisova, N. V.; Chernov. A. N.; Pushin, A. N.; Zefirov, N. S. Zh. Org. Khim. 1992, 28, 934. (d) Gritsenko, E. I.; Butenko, G. G.; Plementov, V. V.; Balueva, A. I.; Bolesov, I. G. J. Gen. Chem. USSR (Engl. Trans.) 1986, 56, 561.

9. For carbocupration see: (a) Nakamura, E.; Isaka, M.; Matsuzawa, S. J. Am. Chem. Soc. 1988, 110, 1297. (b) Isaka, M.; Nakamura, E. J. Am. Chem. Soc. 1990, 112, 7428. (c) Nakamura, E.; Kubota, K.; Isaka, M. J. Org. Chem. 1992, 57, 5809. (d) Kubota, K.; Isaka, M.; Nakamura, E. Heterocycles 1996, 42, 565. For allylzincation see: (e) Kubota, K.; Nakamura, M.; Isaka, M.; Nakamura, E. J. Am. Chem. Soc. 1993, 115, 5867. (f) Nakamura, M.; Arai, M.; Nakamura, E. J. Am. Chem. Soc. 1995, 117, 1179. For addition of Grignard reagents see: (g) Nesmeyanova, O. A.; Rudashevskaya, T. Y.; Dyachenko, A. I.; Savilova, S. F.; Nefedov, O. M. Synthesis 1982, 296. (h) Richey, H. G., Jr.; Bension, R. M. J. Org. Chem. 1980, 45, 5036. For mercuriation see: (i) Kartashov, V. R.; Skorobogatova, E. V.; Malisova, N. V.; Grishin, Y. K.; Bazhenov, D. V.; Zefirov, N. S. Zh. Org. Khim. 1991, 27, 2490 .

10. (a) Padwa, A.; Wannamaker, M. W. Tetrahedron 1991, 47, 6139. (b) Padwa, A.; Wannamaker, M. W.; Dyszlewski, A. D. J. Org. Chem. 1987, 52, 4760. (c) Shapiro, E. A.; Protopopova, M. N.; Nefedov, O. M. Izv. Akad. Nauk SSSR, Ser. Khim. 1991, 220. (d) Shapiro, E. A.; Kalinin, A. V.; Nefedov, O. M. Org. Prep. Proc. Int. 1992, 24, 517.

11. Doyle, M. P.; Protopopova, M.; Müller, P.; Ene, D.; Shapiro, E. A. J. Am. Chem. Soc. 1994, $116,8492$.

12. Imogaï, H.; Bernardinelli, G.; Gränicher, C.; Moran, M.; Rossier, J.-C.; Müller, P. Helv. Chim. Acta 1998, 81, 1754.

13. (a) Doyle, M. P.; van Leusen, D. J. Org. Chem. 1982, 47, 5326. (b) Wenkert, E.; Greenberg, R. S.; Raju, M. S. J. Org. Chem. 1985, 50, 4681. (c) Marino, J. P.; Laborde, E. J. Org. Chem. 1987, 52, 1. (d) Brückner, C.; Reissig, H.-U. J. Org. Chem. 1988, 53, 2440. 